\title{
Bilateral Type 1 Idiopathic Macular Telangiectasia in a Female Patient: Multimodal Imaging of a Rare Presentation
}

\author{
Mohamad Reza Niyousha $^{a} \quad$ Narges Hassanpoor $^{a}$ Sasan Jafarib \\ aRetina \& Vitreous Service, Nikookari Eye Hospital, Tabriz University of Medical Sciences, \\ Tabriz, Iran; ${ }^{b}$ Ophthalmology Resident, Nikookari Eye Hospital, Tabriz University of Medical \\ Sciences, Tabriz, Iran
}

\section{Keywords}

Idiopathic macular telangiectasia type $1 \cdot$ Mac tel $\cdot$ Telangectasia $\cdot$ Bilateral $\cdot$ Female $\cdot$ Optical coherence tomography angiography

\begin{abstract}
We report a bilateral case of type 1 idiopathic macular telangiectasia (IMT) in a female patient. A 40-year-old otherwise healthy female with gradual vision loss since 2 years ago with bestcorrected visual acuity of 20/400 in both eyes was referred. Her past medical history was negative for any systemic disease including diabetes and systemic hypertension. Color fundus photography showed bilateral loss of normal foveal reflex with macular edema. Fluorescein angiography demonstrated symmetric perifoveal telangiectasia mainly in the superior and nasal macula in both eyes with late staining and leakage. Spectral-domain optical coherence tomography revealed significant intraretinal fluid bilaterally and subretinal fluid in the left eye. Optical coherence tomography angiography (OCTA) revealed obvious saccular parafoveal capillary telangiectasia and capillary dropout as well as decreased vascular density in both superficial and deep capillary plexus. Deep capillary plexus involvement in OCTA was more evident than superficial plexus. Based on the patient's medical history and multimodal imaging, the diagnosis of bilateral IMT type 1 was made. The patient underwent 5 intravitreal monthly injection of bevacizumab in both eyes, which resulted in macular edema resolution. However, after 3 months of discontinuation of intravitreal bevacizumab, macular edema relapsed. In conclusion, type 1 IMT can occur bilaterally in an otherwise healthy female patient as a very rare presentation. To the best of our knowledge, this case is the 4th case of bilateral type 1 IMT reported in a female.
\end{abstract}


Niyousha et al.: Bilateral IMT Type 1 in a Female

\section{Introduction}

Idiopathic macular telangiectasia (IMT) is a retinal vascular disease classified by Gass and Blodi [1]. On the basis of biomicroscopic and fluorescein angiographic features, 3 general types have been described. IMT type 1 is an aneurysmal telangiectasia mainly affecting men unilaterally and is divided into 2 subtypes: type $1 \mathrm{~A}$ is characterized by prominent telangiectatic retinal capillaries usually at the temporal half of the macula that involves 2 disc diameters or more. Vascular leakage, cystoid macular edema, and yellowish exudation as a variant of Coats' disease can be seen. More than $90 \%$ of patients are males with unilateral involvement. Type 1B also shows similar male preference, unilaterality, and biomicroscopic and fluorescein angiographic features, and the only difference is that the extent of telangiectatic involvement in retinal capillaries is less than IMT type 1A.The severity of disease and visual impairment is also milder [1].

More recently, Yannuzzi et al. [2] merged the type 1A and 1B subgroups into type 1 aneurysmal telangiectasia [3]. Distribution of retinal involvement is varied from vascular lesions in the para-macular or juxtafoveal area to focal vascular changes in the mid-periphery and even in anterior retina. IMT type 1 is usually considered to be a form of Coats' disease. Optical coherence tomography shows macular edema and foveal exudative cystic changes. On fluorescein angiography (FA), telangiectatic vessels are visible, filling promptly in both superficial and deep juxtafoveolar capillary plexus. Minimal non-perfusion or capillary ischemia and cystic or non-cystic macular edema as late intraretinal staining and leakage can also be seen $[2,4,5]$.

As we mentioned, type 1 IMT is unilateral parafoveal telangiectasia that typically occurs in males. We report a case of a 40-year-old woman with bilateral type 1 IMT. To the best of our knowledge, this case is the 4th case of bilateral type 1 IMT reported in a middle-aged healthy female $[2,3]$.

\section{Case Report}

A 40-year-old woman referred to our clinic complaining of gradual vision loss in her both eyes since about 2 years ago. Her best-corrected visual acuity was 20/400 in both eyes. Her past medical history was unremarkable. Anterior segment examination had no significant point. Color fundus photography showed bilateral loss of normal foveal reflex with macular edema. Moreover, hard exudates could be seen in the inferotemporal retina of the right eye. Early- and late-phase FA (Spectralis, Heidelberg Engineering, Heidelberg, Germany) showed perifoveal telangiectasia and microaneurysms mainly in the superior and nasal area of macula in both eyes with late staining and leakage. Blue-wavelength autofluorescence showed bilateral abnormal hyper-autofluorescence areas due to cystoid macular edema. FA and blue-wavelength autofluorescence showed highly symmetric changes in both eyes (Fig. 1, 2).

Spectral-domain optical coherence tomography (Spectralis Heidelberg Engineering, Heidelberg, Germany) demonstrated significant intraretinal fluid bilaterally and subretinal fluid in the left eye. Central macular thickness measured up to $721 \mu \mathrm{m}$ on the right eye and $673 \mu \mathrm{m}$ on the left eye. Optical coherence tomography angiography (OCTA; Optovue, Inc, Fremont, CA, USA) was applied for this patient. OCTA revealed obvious saccular parafoveal capillary telangiectasia, capillary dropout and decreased vascular density in both superficial and deep capillary plexus. Deep capillary plexus involvement in OCTA was more than superficial plexus. Both superficial and deep capillary plexus involvement crossed the horizontal line based on OCTA (Fig. 3).

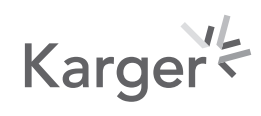



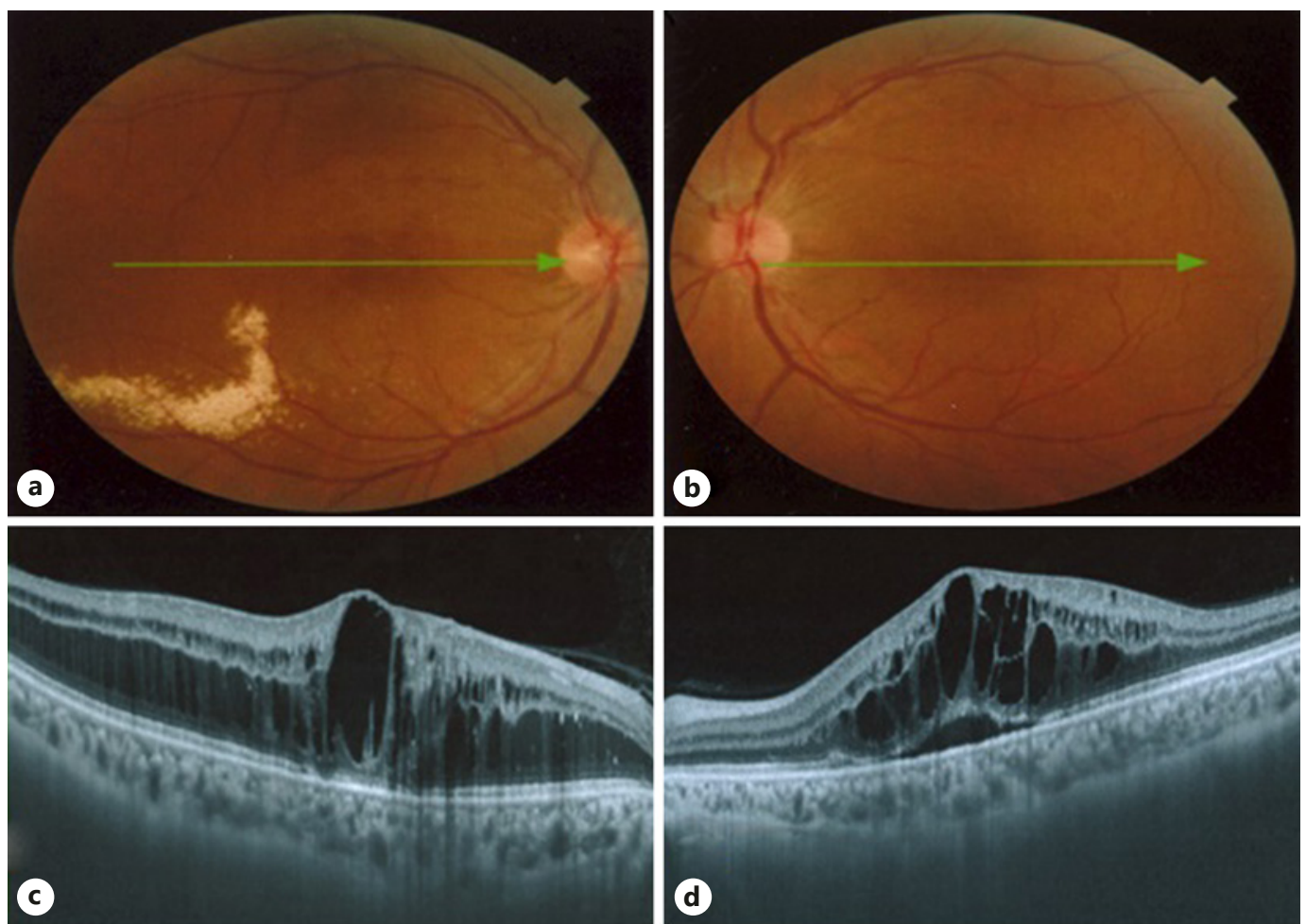

c
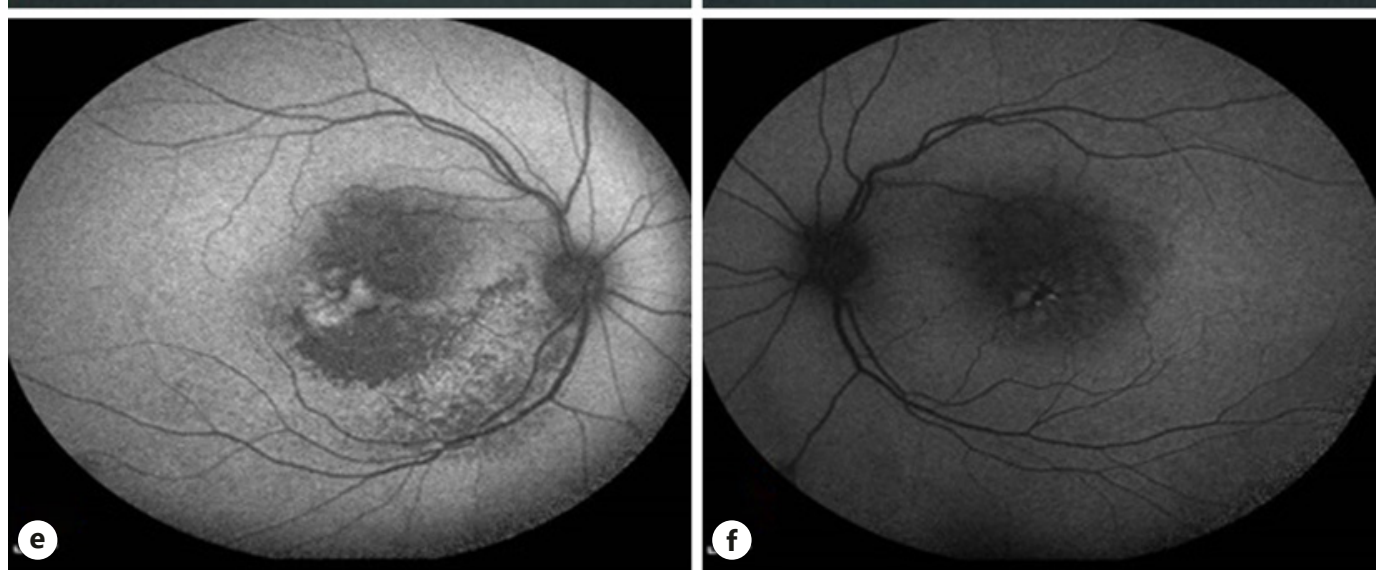

Fig. 1. Color fundus photography $(\mathbf{a}, \mathbf{b})$ shows bilateral loss of normal foveal reflex. Some hard exudates could be seen in the inferotemporal retina of the right eye. SD-OCT (c, d) demonstrates significant intraretinal fluid bilaterally and mild subretinal fluid in the left eye. Autofluorescence (e, f) shows bilateral loss of normal foveal hypo-autofluorescence, replaced by petaloid hyper-autofluorescence areas due to cystoid edema. SD-OCT, spectral-domain optical coherence tomography.

The patient had no history of hypertension, diabetes, hematologic abnormality and other systemic diseases or X-ray radiation. Her familial, past ocular, and past drug history were also negative. Based on multimodal imaging, diagnosis of bilateral IMT type 1 was made. The patient was treated with intravitreal injection of bevacizumab for 5 times. Macular edema improved significantly by this treatment. However, macular edema and vision loss relapsed after 3 months of discontinuation of intravitreal bevacizumab. Injections restarted but after discontinuing the injections due to patient poor compliance, macular edema relapsed again. Therefore, macular photocoagulation was applied in involved areas based on FA and OCTA with good response in both eyes (Fig. 4). 


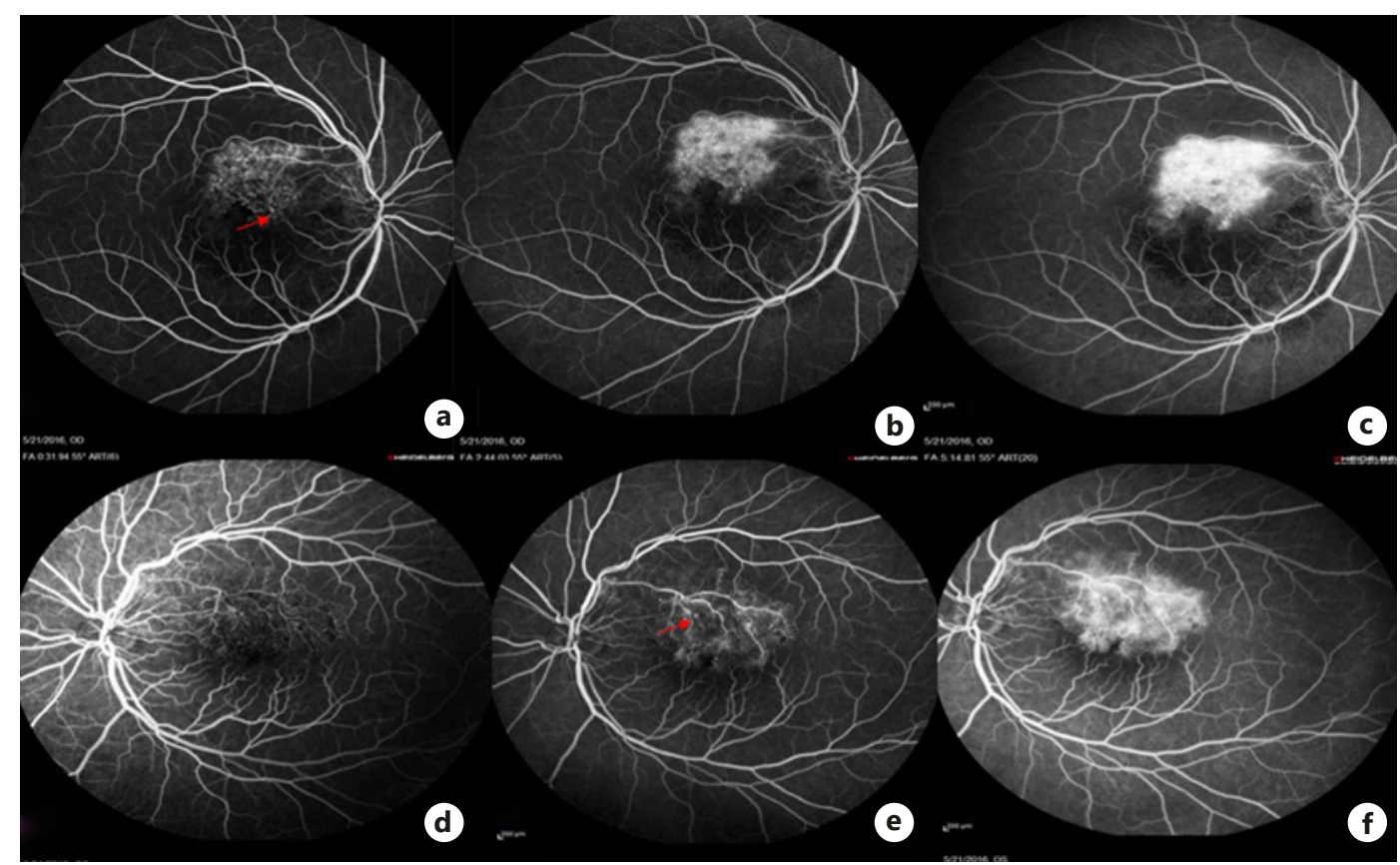

Fig. 2. Early- and late-phase fluorescein angiography (a-f) shows perifoveal telangiectasia, microaneurysms (red arrows), and macular edema mainly in the superior and nasal of fovea in both eyes with late staining and leakage. Note that lesions are highly symmetric.

\section{Discussion}

IMT type 1 is characterized by unilateral macular telangiectasia and aneurysms typically in males. Bilateral presentation in a female patient is very rare. The possible disorders that can cause similar presentation are retinopathies caused by diabetes mellitus, sickle cell anemia, hypertension, retinal venous occlusions, ischemic syndromes, hyper-coagulopathy, inflammation, and retinal hemangiomas. The past medical history of this patient was negative. Therefore, none of these disorders can be attributed to our patient.

The most important differential diagnosis of this condition is bilateral branch retinal vein occlusion (BRVO). However, we should notice some important points before the diagnosis is made. First, bilateral cases of BRVO are often related to systemic diseases especially in young patients [6]. Second, in IMT1, retinal hard exudates can be seen far away from leaking areas (as it is obvious in our patient, Fig. 1a), far to consider it due to BRVO. It senses like a Coats phenomenon that exudate is far from pathologic area [7].

Third, natural course of IMT1 is somehow different from BRVO that usually we have stable course after macular edema resolution by multiple intravitreal injections [8,9]. Our patient showed multiple recurrences early after discontinuation of intravitreal injections and became stable after laser photocoagulation treatment. The limited effect of intravitreal antiVEGF treatment for IMT type 1 and need to use focal laser treatment as the mainstay in controlling macular edema has been focused earlier in literature [10,11]. Although there are some recurrent BRVO cases in the literature, we cannot rule out this diagnosis only based on multiple recurrences.

Fourth point and probably the most important point is similarity between the 2 eyes. In this patient, location and pattern of involvement is exactly the same for both eyes, simulating a kind of dystrophy rather an acquired and random condition like BRVO [2,3] (Fig. 2).

\section{Karger'}



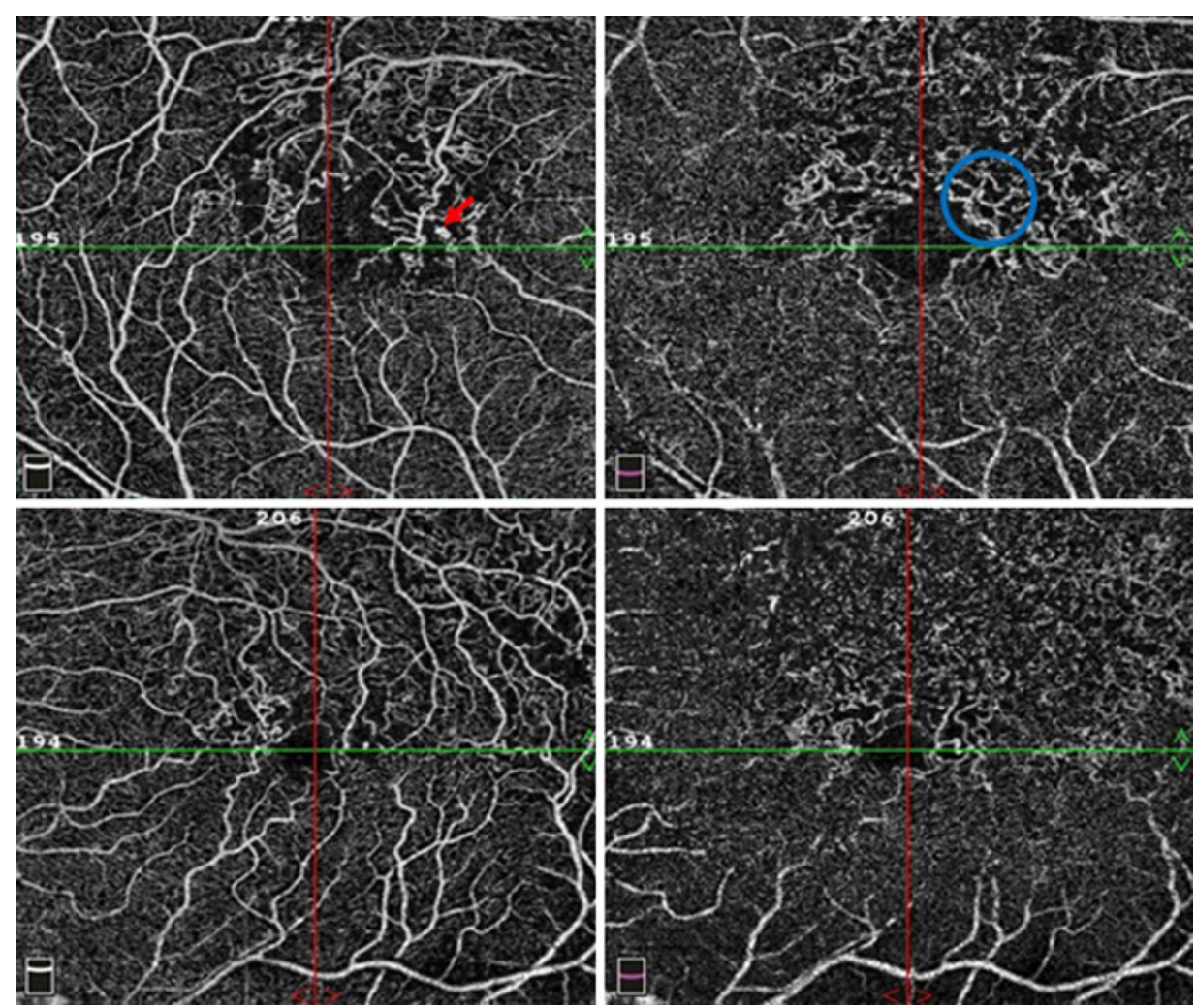

Fig. 3. OCTA revealed obvious saccular parafoveal capillary telangiectasia (red arrow), increased vascular tortuosity (blue circle), capillary dropout, and decreased vascular density in both superficial (left column) and deep (right column) capillary plexus. Deep capillary plexus involvement in OCTA was more than superficial plexus. Both superficial and deep capillary plexus involvement crossed the horizontal line based on OCTA. OCTA, optical coherence tomography angiography.

Fifth, both superficial and deep capillary plexus involvement crossed the horizontal line based on OCTA. This phenomenon is considered to be in favor of IMT type 1 rather than BRVO [10].

We described a case of atypical bilateral type 1 IMT in a female patient. This presentation is unusual for type 1 IMT and has only been described rarely in literature. Usual cases occur unilaterally with male predominance. In a similar study in 2017, by Machkour et al. [3], 2 cases of middle-aged women with bilateral type 1 IMT have been described. In an older study in 2006 by Yannuzzi et al. [2], another case of bilateral IMT in a female has been described. To the best of our knowledge, this case is the 4th case of bilateral type 1 IMT reported in a middle-aged healthy female.

In Gass and Blodi's study [1], telangiectasia was present bilaterally in 1 male case among 8 patients of IMT. Finally, Dunn et al. [5] also reported a male patient with bilateral type 1 IMT complicated by cystoid macular edema.

As mentioned, type 1 IMT is a variant of Coats disease. Coats and type 1 IMT predominantly affect males and are usually unilateral. As we know, Coats disease is more unusual, advanced, and atypical in female gender and bilaterality can occur more in female patients. As most cases of bilateral IMT reported to date are female, this suggests same pattern in type 1 IMT patients. In this case, we also tried to show that OCTA may effectively identify vascular anomalies in type 1 IMT patients and be useful as a guide for laser treatment. 


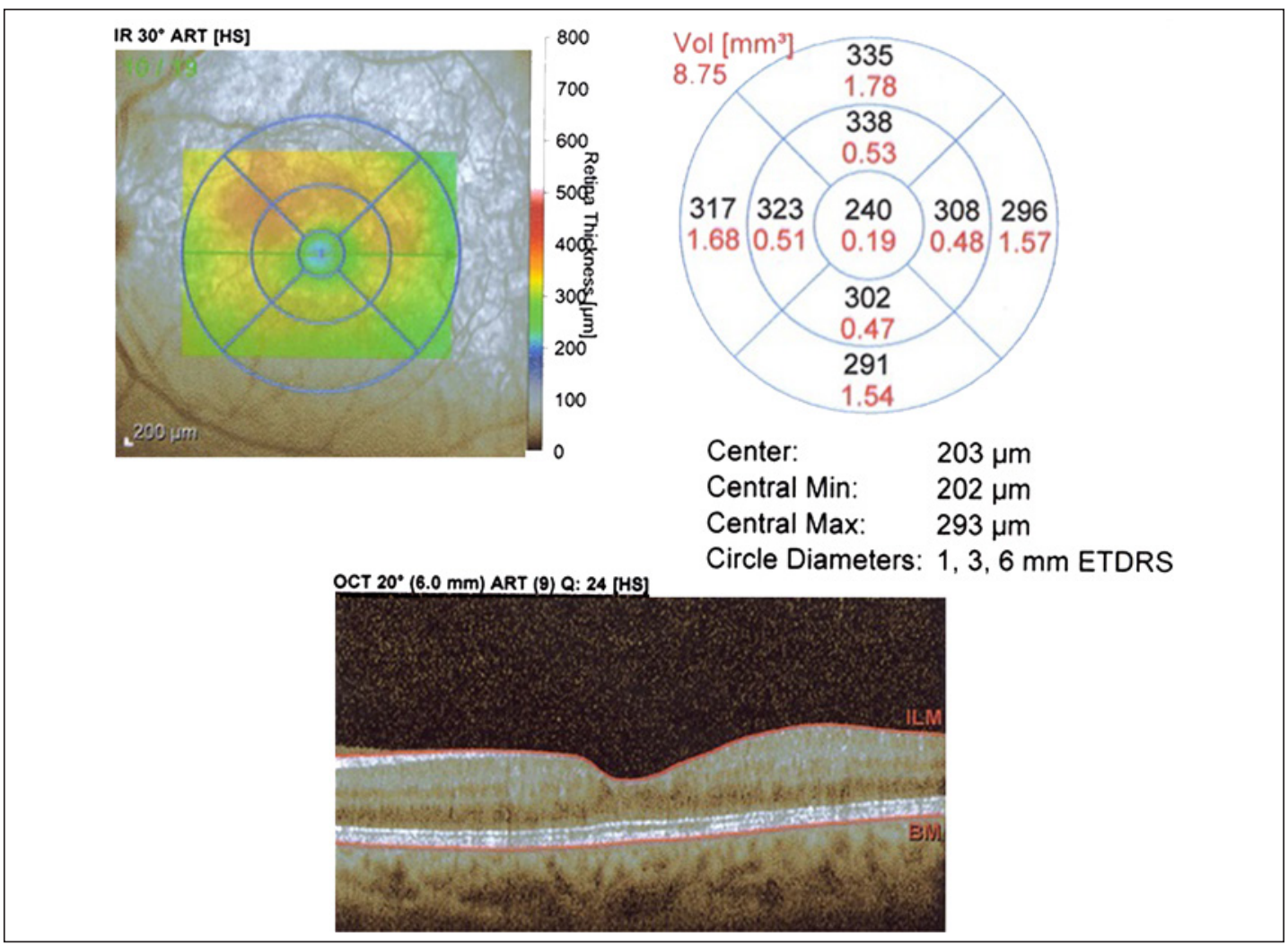

Fig. 4. Post-laser treatment OCT of the patient. OCT, optical coherence tomography.

\section{Conclusion}

According to this case and previous studies [1-3, 5], it seems bilateral involvement and female sex cannot rule out the diagnosis of type 1 IMT. Moreover, typical features of type 1 IMT in fundus examination may not be seen in all cases and we should consider other modalities like FA and OCTA alongside fundus examination for making the proper diagnosis. Further, similar case reports in the future may help us to figure out new pathophysiologic and clinical aspects of type 1 IMT.

\section{Statement of Ethics}

Written informed consent was acquired from the patient to report her clinical materials. This study adhered to the tenets of the Declaration of Helsinki.

\section{Conflict of Interest Statement}

The authors have no conflicts of interest to declare.

\section{Funding Sources}

The authors did not receive any funding.

\section{Karger'}


Niyousha et al.: Bilateral IMT Type 1 in a Female

\section{Author Contributions}

All authors contributed in patient evaluation and manuscript writing and approved the final version of the manuscript for publication.

\section{References}

1 Gass JD, Blodi BA. Idiopathic juxtafoveolar retinal telangiectasis. Update of classification and follow-up study. Ophthalmology. 1993;100(10):1536-46.

2 Yannuzzi LA, Bardal AM, Freund KB, Chen KJ, Eandi CM, Blodi B. Idiopathic macular telangiectasia. 2006. Retina. 2006;32(Suppl 1):450-60.

3 Machkour ZB, Denis P, Kodjikian L. Unusual presentation of type 1 idiopathic macular telangiectasia. Case Rep Ophthalmol Med. 2017;2017:5395069.

4 Nowilaty SR, Al-Shamsi HN, Al-Khars W. Idiopathic juxtafoveolar retinal telangiectasis: a current review. Middle East Afr J Ophthalmol. 2010;17(3):224-41.

5 Dunn EN, Gregori NZ, Goldhardt R. Phakic cystoid macular edema secondary to idiopathic macular telangiectasia type 1 responsive to topical anti-inflammatory agents. Semin Ophthalmol. 2013 Mar;28(2):84-7.

6 Castro-Navarro V, Odaibo SG, Ghodasra DH, Besirli CG. Bilateral BRVO in a patient with recurrent prostate cancer. BMJ Case Rep. 2015 Oct 21;2015:bcr2015212463.

7 Rehak J, Rehak M. Branch retinal vein occlusion: pathogenesis, visual prognosis, and treatment modalities. Curr Eye Res. 2008 Feb;33(2):111-31.

8 Hikichi T, Higuchi M, Matsushita T, Kosaka S, Matsushita R, Takami K, et al. Two-years outcomes of intravitreal bevacizumab therapy for macular oedema secondary to branch retinal vein occlusion. Br J Ophthalmol. 2014; 98(2):195-9.

9 Rush RB, Simunovic MP, Aragon AV 2nd, Ysasaga JE. Treat-and-extend intravitreal bevacizumab for branch retinal vein occlusion. Ophthalmic Surg Lasers Imaging Retina. 2014;45(3):212-6.

10 Osaka R, Shiragami C, Ono A, KobayashiTakasago MY, Takasago Y, Yamashita A, et al. Clinical features of treated and untreated type 1 idiopathic macular telangiectasia without the occurrence of secondary choroidal neovascularization followed for 2 years in Japanese patients. Retina. 2018 Jan;38(Suppl 1):S114-22.

11 Chatziralli IP, Sharma PK, Sivaprasad S. Treatment modalities for idiopathic macular telangiectasia: an evidence-based systematic review of the literature. Semin Ophthalmol. 2017;32(3):384-94. 